\title{
Recommendations on the Diagnosis and Initial Management of Acute Variceal Bleeding and Hepatorenal Syndrome in Patients with Cirrhosis
}

\author{
Frederik Nevens ${ }^{1}\left[\right.$ (D) Paulo Lisboa Bittencourt ${ }^{2} \cdot$ Minneke J. Coenraad ${ }^{3} \cdot$ Huiguo Ding $^{4} \cdot$ Ming-Chih Hou $^{5}$. \\ Pierre-François Laterre $^{6} \cdot$ Manuel Mendizabal $^{7} \cdot$ Nayeli Xochiquetzal Ortiz-Olvera $^{8} \cdot$ Julio D. Vorobioff $^{9}$. \\ Wenhong Zhang ${ }^{10} \cdot$ Paolo Angeli ${ }^{11}$
}

Received: 20 April 2018 / Accepted: 29 December 2018 / Published online: 25 January 2019

๑) Springer Science+Business Media, LLC, part of Springer Nature 2019

\begin{abstract}
Cirrhosis is a serious and life-threatening condition which imposes a significant socioeconomic burden on affected individuals and healthcare systems. Cirrhosis can result in portal hypertension, which may lead to major complications, including acute variceal bleeding and hepatorenal syndrome. Without prompt treatment, these complications may be life-threatening. Over the past 2 decades, new treatment modalities and treatment strategies have been introduced, which have improved patients' prognosis, but the initial management of these severe complications continues to present a challenge. The present recommendations aim to increase clinicians' knowledge on the importance of early diagnosis and treatment, and to provide evidence-based management strategies to potentially, further improve patient outcomes. Special attention was given to the role of terlipressin. A comprehensive non-systematic literature search was undertaken to evaluate the evidence for the diagnosis and initial management of acute variceal bleeding and hepatorenal syndrome in patients with cirrhosis. Recommendations on the diagnosis and initial management of acute variceal bleeding and hepatorenal syndrome in patients with cirrhosis have been developed based on the best available evidence and the expert opinion of the consensus panel following a comprehensive review of the available clinical data. Prompt identification and timely treatment of acute variceal bleeding and hepatorenal syndrome are essential to reduce the burden.
\end{abstract}

Keywords Acute variceal bleeding $\cdot$ Acute kidney injury $\cdot$ Hepatorenal syndrome $\cdot$ Cirrhosis $\cdot$ Terlipressin $\cdot$ Vasoactive drugs

\section{Introduction}

Cirrhosis is a serious and life-threatening condition which imposes a significant socioeconomic burden on affected individuals and healthcare systems worldwide [1,2]. According to a population-based study undertaken in the USA, cirrhosis affects over 633,000 adults each year [3] and represents the sixth most common cause of death due to non-communicable diseases worldwide [4]. In Europe, cirrhosis is responsible for 170,000 deaths each year, with wide variations between countries [5]. Many cases may be undiagnosed, and more than half are potentially preventable [3].

Frederik Nevens

frederik.nevens@uzleuven.be

Extended author information available on the last page of the article
Cirrhosis can result in portal hypertension, which may lead to major complications including bleeding gastroesophageal varices and hepatorenal syndrome (HRS), a severe form of acute kidney injury (AKI) that usually presents in patients with decompensated liver cirrhosis. Esophageal varices are present in 30-70\% of patients with cirrhosis at the time of diagnosis and tend to increase in size in a linear fashion [6, 7]. Approximately $4-30 \%$ of patients with small varices will develop large varices each year and are at risk of bleeding [6]. Gastric varices are less common than esophageal varices, occurring in $5-33 \%$ of patients with portal hypertension [6], but bleeding tends to be more severe, with a reported mortality of approximately $45 \%$ [8]. In addition, a high proportion of patients with gastric varices rebleed after spontaneous hemostasis [9].

Acute variceal bleeding can be fatal and remains a major public health problem. In the early 1980s, in-house mortality 
after variceal hemorrhage was around $40 \%$ [10]; however, in recent years, in-hospital mortality has fallen steadily to $10-20 \%$, due to new knowledge on the pathophysiology of acute variceal bleeding and advances in therapy [11-13]. Although mortality has decreased, bleeding esophageal varices (BEV) still remain of significant clinical importance. The risk of rebleeding, which is most likely to occur within 6 weeks of the original episode, remains appreciably high [14]. In a nationwide audit undertaken in the UK, mortality in those patients who suffered from rebleeding was more than fivefold (39 vs. 7\%) greater than those patients who did not rebleed [14].

HRS is a potentially reversible form of AKI that usually presents in patients with decompensated liver cirrhosis. Between 20 and $50 \%$ of hospitalized patients with cirrhosis have AKI [15]. HRS is classified into two types based on the clinical course, with type 1 HRS being the predominant type [16]. Type 1 HRS is defined by a rapidly progressive reduction of renal function in less than 2 weeks, while type $2 \mathrm{HRS}$ is a less progressive, moderate course of renal failure characterized by a serum creatinine ( $\mathrm{sCr}$ ) level of $>1.5 \mathrm{mg}$ / $\mathrm{dL}(>133 \mu \mathrm{mol} / \mathrm{L})$ and is usually accompanied by refractory ascites $[17,18]$. In type $1 \mathrm{HRS}$, a precipitating factor frequently is identified, whereas type 2 HRS arises spontaneously [18]. The prognosis of patients with HRS is poor, with a reported median survival after diagnosis of only 1.7 weeks [19]. Evidence suggests, however, that HRS is potentially reversible if hemodynamic derangements are corrected in a timely fashion [20].

There have been significant advances in the understanding and treatment of acute variceal bleeding and HRS in patients with cirrhosis, and improved survival, but the initial management of these serious complications continues to present a challenge and requires a multidisciplinary approach. The purpose of these recommendations is to provide clinicians with evidence-based management strategies that will potentially, further improve patient outcomes.

\section{Methods}

A multidisciplinary, consensus panel, comprising gastroenterologists, hepatologists and critical care physicians from Asia, Europe and South America, convened at a meeting in London, UK, in November 2016 to review the available evidence for the diagnosis and initial management of acute variceal bleeding and HRS in patients with cirrhosis and to develop a set of recommendations. A comprehensive, non-systematic search of the relevant literature electronically indexed in PubMed, EMBASE, the Cochrane Library and clinicaltrials.gov was undertaken using the following key words: hepatorenal syndrome, cirrhosis, acute kidney injury, varices, vasoactive, terlipressin, vasopressin, octreotide, midodrine, noradrenaline. No language limits were set on the database searches. Randomized and nonrandomized controlled trials, crossover trials, single-arm studies, cohort studies, case-control studies and case series were included, as well as published data from registries and medical databases. The search results were supplemented by manual searching of relevant journals, reference lists in key journals and other appropriate documents, as well as expert opinion. All recommendations were based on the best available evidence combined with the authors' experiences in managing acute variceal bleeding and HRS in patients with cirrhosis. For each section, recommendations were made and evidence levels graded according to the Oxford Centre for Evidence-Based Medicine Levels of Evidence [21, 22]: Grade A (consistent level 1 studies [systematic review of randomized controlled trials (RCT) and RCT]); Grade B (consistent level 2 or 3 studies or extrapolations from level 1 studies [systematic review of cohort studies, cohort studies, and low quality RCT]); Grade C (level 4 studies [systematic review of case-control studies, case-control studies and case series] or extrapolations from level 2 or 3 studies; Grade D (level 5 studies [expert opinion] or troublingly inconsistent or inconclusive studies at any level).

\section{Management of Bleeding Gastroesophageal Varices}

\section{Diagnosis of Varices}

Current guidelines recommend that all patients with cirrhosis should be screened for the presence of varices at the time of diagnosis, with follow-up every $2-3$ years for compensated patients without varices (depending upon liver disease severity) and 1-2 yearly for compensated patients with small varices, to evaluate progression of varices and the need for prophylactic treatment [12, 23]. During follow-up of patients with chronic liver disease, noninvasive methods include transient elastography and use of laboratory and radiological findings, which are useful for the selection of patients for endoscopy [23]. Variceal hemorrhage should be suspected when a patient with known cirrhosis or evidence of portal hypertension presents with upper gastrointestinal hemorrhage [8].

\section{Recommendation}

- All patients with suspicion of cirrhosis should be screened for the presence of gastroesophageal varices (Grade A).

- Noninvasive methods such as elastography are useful to select patients with chronic liver disease for endoscopy to rule out gastroesophageal varices (Grade C). 


\section{Initial Treatment}

Prophylactic measures such as non-selective beta blocker (NSBB) use and endoscopic variceal ligation are available to prevent initial bleeding $[12,23]$. Acute variceal hemorrhage is a medical emergency that requires prompt treatment and should ideally be managed in an intensive care unit. The key goals of treatment are to control the acute hemorrhage, prevent early (within 5 days) rebleeding and reduce mortality. The optimal management of acute bleeding requires a multifactorial approach. Volume replacement should be initiated as soon as possible to restore and maintain hemodynamic stability [24]. The treatment of acute variceal bleeding includes antibiotic prophylaxis, the use of vasoactive drugs and endoscopic treatment.

\section{Antibiotic Prophylaxis}

Bacterial infections are common due to gut translocation and motility disturbances [6]. Standard practice is to give shortterm (maximum 7 days) antibiotic prophylaxis in any patient with cirrhosis and bleeding esophageal varices (BEV) [6, $23,24]$. In a meta-analysis of 5 trials (534 patients), shortterm (5-10 days) antibiotic prophylaxis was shown to significantly increase the mean percentage of patients free of infection (32\% mean improvement rate, 95\% confidence interval $[\mathrm{CI}] 22-42 ; P<0.001)$ and significantly increase short-term survival rate $(9.1 \%$ mean improvement rate, $95 \%$ CI 2.9-15.3; $P=0.004)$ [25].

\section{Vasoactive Drugs}

After resuscitation, volume replacement and prophylactic antibiotics, there are a number of specific treatment options available to treat BEV. To date, the best evidence for efficacy is vasoactive drugs combined with endoscopic treatment as it achieves better control of acute bleeding than endoscopic treatment alone [26]. In a meta-analysis of 8 trials involving 939 patients, combined use of endoscopic and drug therapy improved initial control of bleeding (relative risk [RR] 1.12, 95\% CI 1.02-1.23) and 5-day hemostasis (RR 1.28, 95\% CI 1.18-1.39) [26].

A number of vasoactive drugs, including vasopressin, terlipressin, somatostatin and octreotide, are available. In a meta-analysis of 30 RCTs, the use of intravenously administered vasoactive drugs was shown to significantly lower the risk of all-cause mortality within 7 days (RR $0.74,95 \%$ CI $0.57-0.95 ; P=0.02$ ) and significantly improve hemostasis (RR 1.21, 95\% CI 1.13-1.30; $P<0.001$ ) compared with control. Studies comparing different vasoactive drugs did not show a difference in efficacy, although the quality of evidence was low [27].

Terlipressin is superior to placebo in the control of variceal hemorrhage [28-30] (Table 1). In addition, terlipressin has shown to have mortality benefit in placebocontrolled trials [28, 31] and meta-analysis [32]. Seven studies comparing terlipressin to placebo (443 patients) were included in the meta-analysis by Ioannou et al. [32]. Terlipressin was associated with a statistically significant reduction in all-cause mortality compared with placebo (RR 0.66, 95\% CI 0.49-0.88). Conversely, in comparative studies of different vasoactive drugs (terlipressin, somatostatin and octreotide) no significant differences were found among them regarding control of hemorrhage or mortality (Table 2) [33-38]. Many of these original studies, however, were conducted more than 20 years ago and are limited by small sample sizes and study designs.

\section{Safety of Terlipressin}

Most studies have shown that vasoactive drugs have a good safety profile, although vasopressin can cause an increase in

Table 1 Terlipressin versus placebo in cirrhotic patients with BEV

\begin{tabular}{|c|c|c|c|c|c|c|c|}
\hline \multirow{2}{*}{$\begin{array}{l}\text { Author } \\
\text { Söderlund et al. [28] }\end{array}$} & \multirow{2}{*}{$\begin{array}{l}\text { Study design } \\
\text { Randomized, double-blind, placebo-controlled }\end{array}$} & \multirow{2}{*}{$\begin{array}{l}\text { Drug } \\
\text { Terlipressin }\end{array}$} & \multirow{2}{*}{$\begin{array}{l}n \\
31\end{array}$} & \multicolumn{2}{|c|}{$\begin{array}{l}\text { Control of bleeding } \% \\
(P \text { value })\end{array}$} & \multicolumn{2}{|c|}{$\begin{array}{l}\text { Mortality \% }(P \\
\text { value })\end{array}$} \\
\hline & & & & 90.3 & $(<0.01)$ & $9.7^{\mathrm{a}}$ & $(0.014)$ \\
\hline & & Placebo & 29 & 58.6 & & $37.9^{\mathrm{a}}$ & \\
\hline \multirow[t]{2}{*}{ Freeman et al. [29] } & \multirow[t]{2}{*}{ Randomized, double-blind, placebo-controlled } & Terlipressin & 15 & 60.0 & (NS) & $20.0^{\mathrm{a}}$ & - \\
\hline & & Placebo & 16 & 37.0 & & $25.0^{\mathrm{a}}$ & \\
\hline \multirow[t]{2}{*}{ Walker et al. [30] } & \multirow{2}{*}{$\begin{array}{l}\text { Single center, prospective, randomized, double- } \\
\text { blind, placebo-controlled }\end{array}$} & Terlipressin & $25^{\mathrm{d}}$ & $100^{\mathrm{b}}$ & $(<0.05)$ & $12.0^{\mathrm{c}}$ & (NS) \\
\hline & & Placebo & $25^{\mathrm{d}}$ & $80.0^{\mathrm{b}}$ & & $32.0^{\mathrm{c}}$ & \\
\hline
\end{tabular}

NS not significant

${ }^{\mathrm{a}}$ At discharge

${ }^{b}$ Within $36 \mathrm{~h}$

${ }^{\mathrm{c}}$ Hospital mortality

${ }^{\mathrm{d}}$ Bleeding episodes (34 patients in total) 
Table 2 Terlipressin versus somatostatin or octreotide in cirrhotic patients with BEV

\begin{tabular}{|c|c|c|c|c|c|c|c|}
\hline \multirow{2}{*}{$\begin{array}{l}\text { Author } \\
\text { Feu et al. [35] }\end{array}$} & \multirow{2}{*}{$\begin{array}{l}\text { Study design } \\
\text { Multicenter, double-blind, randomized }\end{array}$} & \multirow{2}{*}{$\begin{array}{l}\text { Drug } \\
\text { Terlipressin }\end{array}$} & \multirow{2}{*}{$\begin{array}{l}n \\
80\end{array}$} & \multicolumn{2}{|c|}{$\begin{array}{l}\text { Control of bleeding } \\
\%(P \text { value })\end{array}$} & \multicolumn{2}{|c|}{$\begin{array}{l}\text { Mortality } \% \\
(P \text { value })\end{array}$} \\
\hline & & & & 80.0 & (NS) & $16.3^{\mathrm{a}}$ & - \\
\hline & & Somatostatin & 81 & 84.0 & & $16.0^{\mathrm{a}}$ & \\
\hline \multirow[t]{2}{*}{ Walker et al. 36] } & \multirow[t]{2}{*}{ Double-blind, randomized, placebo controlled } & Terlipressin & $53^{\mathrm{g}}$ & 90.6 & - & $20.8^{\mathrm{b}}$ & (NS) \\
\hline & & Somatostatin & $53^{\mathrm{g}}$ & 81.1 & & $20.8^{b}$ & \\
\hline \multirow[t]{3}{*}{ Seo et al. [33] } & \multirow[t]{3}{*}{ Non-blinded, non-inferiority } & Terlipressin & 261 & $86.2^{\mathrm{c}}$ & (NS) & $8.0^{\mathrm{c}}$ & (NS) \\
\hline & & Somatostatin & 259 & $83.4^{\mathrm{c}}$ & & $8.9^{\mathrm{c}}$ & \\
\hline & & Octreotide & 260 & $83.8^{\mathrm{c}}$ & & $8.8^{\mathrm{c}}$ & \\
\hline \multirow[t]{2}{*}{ Abid et al. [34] } & \multirow{2}{*}{$\begin{array}{l}\text { Single center, randomized, double-blind, placebo- } \\
\text { controlled, non-inferiority design }\end{array}$} & Terlipressin & 163 & 96.9 & (NS) & $5.5^{\mathrm{d}}$ & $(\mathrm{NS})$ \\
\hline & & Octreotide & 161 & 99.4 & & $4.3^{\mathrm{d}}$ & \\
\hline \multirow[t]{2}{*}{ Pedretti et al. [37] } & \multirow[t]{2}{*}{ Single center, single-blind, randomized } & Terlipressin $^{\mathrm{e}}$ & 30 & 53.3 & (NS) & 13.3 & (NS) \\
\hline & & Octreotide & 30 & 76.6 & & 10.0 & \\
\hline \multirow[t]{2}{*}{ Silvain et al. [38] } & \multirow[t]{2}{*}{ Multicenter, randomized } & Terlipressin & 41 & $59.0^{f}$ & (NS) & $\begin{array}{l}0.0^{\mathrm{f}} \\
28.0^{\mathrm{b}}\end{array}$ & (NS) \\
\hline & & Octreotide & 46 & $78.0^{\mathrm{f}}$ & & $\begin{array}{l}6.0^{\mathrm{f}} \\
22.0^{\mathrm{b}}\end{array}$ & \\
\hline
\end{tabular}

NS not significant

${ }^{\mathrm{a}} 6$ weeks

${ }^{\mathrm{b}} 30$ days

c5 days

${ }^{\mathrm{d}}$ During the trial

${ }^{\mathrm{e}}$ Combined with transdermal nitroglycerine

${ }^{\mathrm{f}}$ At $12 \mathrm{~h}$

g Bleeding episodes (72 patients in total)

peripheral resistance and reduction in cardiac output, heart rate and coronary blood flow [23]. Terlipressin has a favorable adverse effect profile and is related to fewer cardiovascular side effects when compared to vasopressin due to its preferential binding to V1 receptors [39]. Adverse events (AEs) are mostly cardiovascular and related to vasoconstriction [40]. Side effects include angina pectoris, acute myocardial infarction, bradyarrhythmia, peripheral vascular ischemia, mesenteric ischemia and systemic arterial hypertension [39]. In a meta-analysis by Ioannou et el. [32], there was no significant difference between the terlipressin group and any of the comparison groups in the number of AEs that caused death or withdrawal of medication. Terlipressin should be avoided or used with caution in patients with preexisting cardiovascular disease and in patients over 70 years of age as experience is limited in this patient population.

Patients receiving terlipressin for the treatment of BEV may develop hyponatremia, which is usually reversible after terlipressin withdrawal [41, 42]. The most likely explanation of terlipressin-induced hyponatremia is the agonistic activity on renal V2 vasopressin receptors causing free water retention. The administration of hypotonic fluid strongly favors the development of acute hyponatremia in this situation. In two of the studies [41, 42], patients who developed hyponatremia had less advanced liver disease and higher baseline serum sodium concentration, suggesting that in these patients the V2 vasopressin receptors were not yet occupied by endogenous vasopressin. This may explain why hyponatremia is uncommon during the administration of terlipressin for HRS, a situation which occurs in more advanced liver disease. These findings highlight the need for daily monitoring of serum sodium levels in patients receiving terlipressin for acute gastrointestinal bleeding due to portal hypertension, as is routinely performed in all patients with cirrhosis and that in patients with low MELD (Model For End-Stage Liver Disease), and normal to near-normal baseline serum sodium concentrations, hypotonic fluids should be avoided. Whether the administration of albumin might counteract this side effect needs to be explored.

NSBBs should be avoided when treating patients with active BEV with terlipressin since they can potentially induce systemic hypotension [43]. After review of the literature, it is recommended that NSBBs are suspended in patients with BEV during terlipressin infusion and restarted when the terlipressin course is finished. 


\section{Co-administration of Terlipressin and Somatostatin or Octreotide}

Kalambokis et al. [44] evaluated the effects of somatostatin, terlipressin and combined treatment with somatostatin and terlipressin on portal hemodynamics in a randomized, placebo-controlled study of 24 patients with cirrhosis and esophageal varices. Combined treatment did not exert an additive portal hypotensive effect as compared to terlipressin alone. A previous study by Lin et al. [45] also showed a lack of an additive portal hypotensive effect of octreotide and terlipressin in patients with cirrhosis.

\section{Rationale for the Early Use of Vasoactive Drugs}

A prospective cohort study of 314 patients with cirrhosis and esophageal varices (173 [55\%] with no previous history of variceal bleeding) found that about a quarter of deaths occurred very early after bleeding onset, confirming the need for rapid specific management [46]. Available evidence suggests that early use of vasoactive drugs improves hemostasis before endoscopy [31, 47, 48]. In a prospective, randomized study comprising 76 patients with cirrhosis and active upper gastrointestinal bleeding, terlipressin combined with glyceryl trinitrate administered early (before hospital admission) significantly improved the control of bleeding $(P=0.034)$ and reduced mortality at day $15(P=0.035)$ and day $42(P=0.06)$ compared with placebo [31]. In a double-blind, prospective study involving 205 patients, at endoscopy, active bleeding from esophageal varices was less frequent $(P=0.012)$ and the sclerotherapy procedure was easier $(P=0.0027)$ in the somatostatin than in the placebo group [47].

\section{Optimal Dosage Regimen of Terlipressin}

The current standard practice is to give terlipressin as an intravenous injection, initially a $2 \mathrm{mg}$ bolus injection, then 1-2 mg every 4 or $6 \mathrm{~h}$ depending on the bodyweight of the patient and their co-morbidities. Escorsell et al. [49] showed that the hemodynamic effect of terlipressin on portal pressure lasts no more than 3-4 h, which raises the question as to whether continuous infusion may have better efficacy than bolus injections. While a continuous infusion is possible, it remains unclear whether or not it should be considered in clinical practice. A clinical trial comparing the safety and efficacy of the two infusion types in patients with BEV is currently underway [50]. In another study, Chang et al. [51] investigated whether a high-dose terlipressin could control acute variceal hemorrhage more effectively than a low-dose terlipressin. Complete control of bleeding during $24 \mathrm{~h}$ of drug infusion was achieved in $53 \%$ of patients receiving high-dose terlipressin ( $2 \mathrm{mg}$ ) and in $48 \%$ of those treated with low-dose terlipressin $(1 \mathrm{mg})$. Further randomized studies are needed to evaluate the effects of low-dose terlipressin.

Current guidelines recommend that vasoactive drug treatment should be maintained for up to 5 days since this is the time period in which rebleeding is more frequent $[12,23]$. Azam et al. [52] investigated whether terlipressin can be administered for a shorter period of time to patients with BEV. Terlipressin given for $24 \mathrm{~h}$ was shown to be as effective as a 72-h course when used as an adjunctive therapy to successful endoscopic variceal band ligation.

\section{Recommendations}

- If variceal bleeding is suspected, vasoactive drugs should be given before endoscopy (Grade B). Terlipressin is a drug of choice and should be started as soon as possible when available, and there is no contraindication (Grade A).

- The use of continuous infusion of terlipressin in BEV has not been established (Grade D).

- Close monitoring is required in patients with cardiovascular comorbidity taking terlipressin, and a lower dose should be considered. Serum sodium levels should be monitored and the use of hypotonic fluid avoided (Grade D).

- There is insufficient evidence of the additional effect of co-administration of terlipressin with other vasoactive drugs (Grade D).

\section{Endoscopic Therapy}

Endoscopy should be scheduled in all patients with upper gastrointestinal bleeding. However, the timing is still controversial. There are not enough data to support that endoscopy has to be performed within $<12 \mathrm{~h}$. The endoscopic treatment of choice is band ligation (EBL) $[13,53]$. Erythromycin (250 mg IV 30-120 min) before endoscopy in patients with variceal bleeding significantly improves endoscopic visibility and shortens the duration of the index endoscopy [54]. Recommended treatment with combined vasoactive drugs and endoscopic therapy results in successful 5-day control of bleeding in $85-90 \%$ of cases [55].

\section{Transjugular Intrahepatic Portosystemic Shunt Placement}

Studies have shown that transjugular intrahepatic portosystemic shunt (TIPS) is an extremely useful technique for the treatment of BEV in rescue situations and in high-risk patients. In a RCT of 63 high-risk patients with cirrhosis and BEV who had been treated with vasoactive drugs plus endoscopic therapy, the early use of TIPS was associated with significant reductions in treatment failure and mortality. 
Rebleeding or failure to control bleeding occurred in 14 patients in the pharmacotherapy-endoscopic band ligation (EBL) group compared with 1 patient in the early TIPS group $(P=0.001)$. The 1 -year actuarial survival was $61 \%$ in the pharmacotherapy-EBL group compared with $86 \%$ in the early TIPS group $(P<0.001)$ [13]. High-risk patients were defined as Child-Pugh Class B patients with persistent bleeding at endoscopy and Child-Pugh Class C (score $<14$ ). A post-RCT surveillance study confirms these findings in clinical practice [53]. Patients treated with early TIPS had a much lower incidence of failure to control bleeding or rebleeding than patients receiving drugs and endoscopic therapy ( 3 vs. $15 ; P<0.001$ ). The same was observed for mortality [53]. The value of early TIPS outside high-risk patients is currently being investigated.

\section{Recommendations}

- Band ligation is the endotherapy of choice (Grade A).

- Pre-endoscopy infusion of erythromycin should be considered to avoid aspiration pneumonia (Grade B).

- Early TIPS is the treatment of choice in Child-Pugh Class B patients with persistent bleeding during endoscopy and in Child-Pugh Class C (score $<14)$ patients (Grade A).

\section{Cyanoacrylate Injection Therapy}

There are still limited data on the use of tissue glue cyanoacrylate ( $N$-butyl-2-cyanoacrylate) in the treatment of $\mathrm{BEV}$ in patients with cirrhosis, although in one prospective cohort study of 133 consecutive patients, initial hemostasis was achieved in $94.2 \%$ of patients with active bleeding (95\% CI 85.1-98.5). Overall, early recurrent bleeding occurred in $5.2 \%$ of patients (95\% CI 2.3-10.1) [56]. These findings are supported by the results of another prospective study of 63 patients with cirrhosis admitted with an acute episode of BEV. Bleeding was controlled in almost $75 \%$ of patients during the first 5 days after treatment, and $54 \%$ of patients were free of bleeding at 6 weeks after treatment [57].

\section{Recommendation}

- There are limited data on the use of cyanoacrylate injection therapy in the treatment of BEV in patients with cirrhosis (Grade D).

\section{Management of Acute Gastric Variceal Bleeding}

The optimal treatment for bleeding gastric varices remains unclear as there are few RCTs. General consensus is that the initial management is similar to that of BEV, including the use of prophylactic antibiotics, careful fluid resuscitation with a restrictive transfusion policy and the early administration of vasoactive drugs (terlipressin, somatostatin or a somatostatin analogue $[12,58]$. There are limited data on the efficacy of vasoactive drugs in controlling acute gastric variceal bleeding [36], but general consensus is that terlipressin can be considered for patients with acute gastric variceal bleeding when used alongside glue injection of the varices.

Cyanoacrylate has been successfully used in many countries and is effective in the management of bleeding gastric varices [59-62]. Al-Ali et al. [63] retrospectively reviewed 37 patients with portal hypertension secondary to liver disease and active bleeding treated with cyanoacrylate. Hemostasis was achieved in $95 \%$ of patients. Early rebleeding occurred in only 3 patients $(8 \%)$ during the same hospital admission. However, a Cochrane review undertaken in 2015 concluded that the comparison of cyanoacrylate versus alcohol compounds to treat gastric variceal bleeding remains uncertain due to the very low quality of the evidence [64].

In treatment failures, TIPS is considered the treatment of choice [65, 66]. In a study by Chau et al. [65], 112 consecutive patients with uncontrolled bleeding required emergency TIPS (84 with esophageal varices unresponsive to endoscopic therapy and vasoactive drugs and 28 with gastric varices unresponsive to vasoactive drugs). Variceal bleeding was controlled in all patients after TIPS except for one in each group. During a median follow-up period of 7 months, 20 in the BEV group (24\%) and 8 in the gastric varices group (29\%) developed clinical rebleeding. Most early rebleeding (within 7 days after TIPS) was related to esophageal ulceration secondary to previous sclerotherapy. In another study comprising 58 patients refractory to sclerotherapy and pharmacological treatment, hemorrhage was controlled following salvage TIPS in $90 \%$ of patients. Bleeding persisted in $10 \%$ of patients and recurred in 7\% [66]. There is a place for TIPS in the prevention of gastric variceal rebleeding; a RCT of TIPS versus cyanoacrylate injection concluded that TIPS proved more effective than cyanoacrylate in preventing rebleeding from gastric varices [67].

\section{Recommendations}

- Limited data are available on the efficacy of vasoactive drugs in controlling acute gastric variceal bleeding. Terlipressin can be considered when used alongside glue injection of the varices (Grade D).

- TIPS should be considered in patients who have uncontrolled gastric variceal bleeding refractory to endoscopic therapy and vasoactive drugs and for the prevention of gastric variceal rebleed (Grade C). 


\section{Management of Hepatorenal Syndrome}

\section{Diagnosis of HRS-AKI}

The most widely accepted diagnostic criteria for HRS were published by the International Club of Ascites (ICA) in 1996 [68] and modified in 2007 [17]. In 2015, the ICA proposed a new definition for cirrhosis-related AKI and further modifications of the diagnostic criteria for HRS-related AKI [69]. These revisions were needed because the definition of type 1 HRS did not allow physicians to initiate potentially effective treatment, specifically vasoactive drugs and albumin, until the $\mathrm{sCr}$ increased to $\geq 2.5 \mathrm{mg} / \mathrm{dL}$ [69]. Studies have shown that a higher $\mathrm{sCr}$ at the beginning of treatment leads to a lower probability of treatment response with terlipressin and albumin [70,71].

The ICA define AKI as the increase in $\mathrm{sCr}$ of at least $0.3 \mathrm{mg} / \mathrm{dL}(26 \mu \mathrm{mol} / \mathrm{L})$ within $48 \mathrm{~h}$ or a percentage increase $\mathrm{sCr} \geq 50 \%$ from baseline, which is known, or presumed, to have occurred within the prior 7 days [69]. Once AKI is diagnosed, the stage of AKI should be identified (Table 3).

For patients to be diagnosed with HRS-AKI using these criteria, they need first to progress from Stage 1 to Stage 2 and 3 AKI, when the withdrawal of diuretics is recommended (if they have not been withdrawn already), and volume expansion with albumin $(1 \mathrm{~g} / \mathrm{kg})$ should be administered for 2 days. If the patient then fails to respond, the HRS-AKI criteria should be used to determine whether the patient has HRS-AKI or another AKI phenotype [69]. The ICA criteria for the diagnosis of HRS-AKI are summarized in Table 4. At present, these guidelines do not differentiate between type 1 and type 2 HRS, although there are clear differences between the two and the management of patients differs depending on HRS subtype.

\section{Recommendations}

- It is important to make the diagnosis of HRS-AKI or identify other known causes of renal failure in cirrhosis as early as possible. The diagnosis should be based on the revised criteria for HRS-AKI defined by the ICA (Grade A).

\section{Pharmacological Treatment}

\section{Type 1 HRS}

The general management of patients with cirrhosis and type 1 HRS depends on the severity of kidney failure and associated complications. Once diagnosed, it is recommended that 
Table 4 International Club of Ascites diagnostic criteria of HRS type of AKI in patients with cirrhosis (HRS-AKI) [69]

Diagnosis of cirrhosis and ascites

Diagnosis of AKI according to ICA-AKI criteria

No response after 2 consecutive days of diuretic withdrawal and plasma volume expansion with albumin $1 \mathrm{~g}$ per $\mathrm{kg}$ of body weight

Absence of shock

No current or recent use of nephrotoxic drugs (non-steroidal antiinflammatory drugs, aminoglycosides, iodinated contrast media, etc.)

No macroscopic signs of structural kidney injury ${ }^{\mathrm{a}}$, defined as:

Absence of proteinuria ( $>500 \mathrm{mg} /$ day)

Absence of microhematuria ( $>50$ red blood cells per high power field)

Normal findings on renal ultrasonography

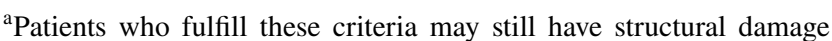
such as tubular damage

treatment is started early in order to prevent the progression of renal failure [72]. The goal of treatment is to improve renal function. The administration of vasoactive drugs (terlipressin, noradrenaline and midodrine plus octreotide) in combination with albumin is currently considered the treatment of choice. Albumin has been shown to improve circulatory function in cirrhosis by expanding central blood volume and increasing cardiac output [73]. Among the vasoactive drugs, terlipressin is the most widely studied for the treatment of type 1 HRS.

In clinical trials and published meta-analyses [20, 74, 75], terlipressin treatment was superior to placebo for achieving reversal of HRS. Meta-analysis of 12 RCTs enrolling 700 patients with type 1 HRS supports the beneficial effect of terlipressin on renal function [20]. Treatment with terlipressin in combination with albumin led to HRS reversal more frequently than albumin alone or placebo (RR 2.54, 95\% CI 1.51-4.26), although the mortality benefit was less clear. Noradrenaline was effective in reversing HRS, but trials were small and non-blinded [20].

Studies have also shown that the administration of terlipressin, together with albumin, is effective in improving renal function in patients with cirrhosis and HRS compared with albumin administration alone [76-80]. In REVERSE [76], a large, phase 3 multicenter, randomized, placebocontrolled trial, which included 196 patients with cirrhosis and type $1 \mathrm{HRS}$, terlipressin plus albumin was more effective than placebo plus albumin in improving renal function, based on a significant decrease in $\mathrm{SCr}$ value from baseline to end of treatment $(P<0.001)$. However, there was no significant difference in the incidence of confirmed HRS reversal (19.6 vs. $13.1 \%$ ) or HRS reversal (23.7 vs. $15.2 \%)$ between the two groups. Further analysis of pooled data from REVERSE and OT-0401, a similarly designed phase 2 clinical study comprising 308 patients, demonstrated that the addition of terlipressin to albumin provides a significant benefit in terms of HRS reversal in patients with type 1 HRS [77].

Other drugs currently used in the management of type 1 HRS include noradrenaline and midodrine, alpha-adrenergic agonists that lead to constriction of vascular smooth muscle and increase systemic vascular resistance [20]. Their effectiveness has been studied in several studies, and data suggest that noradrenaline is as effective as terlipressin in improving renal function in patients with cirrhosis and HRS (Table 5) [81-84]. Midodrine is often used in combination with octreotide. Cavallin et al. [85] compared the effectiveness of terlipressin plus albumin with oral midodrine and

Table 5 Terlipressin versus noradrenaline in patients with type 1 HRS

\begin{tabular}{|c|c|c|c|c|c|c|c|}
\hline \multirow{2}{*}{$\begin{array}{l}\text { Author } \\
\text { Alessandria et al. [84] }\end{array}$} & \multirow{2}{*}{$\begin{array}{l}\text { Study design } \\
\text { Single center, prospective, randomized, non-blinded, pilot }\end{array}$} & \multirow{2}{*}{$\begin{array}{l}\text { Drug }^{\mathrm{d}} \\
\text { Terlipressin }\end{array}$} & \multirow[t]{2}{*}{$n$} & \multicolumn{2}{|c|}{$\begin{array}{l}\text { Reversal of } \\
\text { HRS \% ( } P \\
\text { value) }\end{array}$} & \multicolumn{2}{|c|}{$\begin{array}{l}\text { Survival } \% \\
(P \text { value })\end{array}$} \\
\hline & & & & 80.0 & - & $80.0^{\mathrm{a}}$ & - \\
\hline & & Noradrenaline & 4 & 75.0 & & $50.0^{\mathrm{a}}$ & \\
\hline \multirow[t]{2}{*}{ Sharma et al. [83] } & \multirow[t]{2}{*}{ Single center, open label, pilot, randomized controlled } & Terlipressin & 20 & 50.0 & (NS) & $55.0^{\mathrm{b}}$ & (NS) \\
\hline & & Noradrenaline & 20 & 50.0 & & $55.0^{\mathrm{b}}$ & \\
\hline \multirow[t]{2}{*}{ Singh et al. [82] } & \multirow[t]{2}{*}{ Single center, prospective, randomized, non-blinded } & Terlipressin & 23 & 39.1 & (NS) & $39.1^{\mathrm{b}}$ & $(\mathrm{NS})$ \\
\hline & & Noradrenaline & 23 & 43.4 & & $47.8^{\mathrm{b}}$ & \\
\hline \multirow[t]{2}{*}{ Goyal et al. [81] } & \multirow[t]{2}{*}{ Single center, prospective, open label, randomized } & Terlipressin & 20 & 45.0 & (NS) & $45.0^{\mathrm{c}}$ & - \\
\hline & & Noradrenaline & 21 & 47.6 & & $47.6^{\mathrm{c}}$ & \\
\hline
\end{tabular}

$N S$ not significant

a 180 -day survival

b 15-day survival

c14-day survival

${ }^{\mathrm{d}}$ Albumin was administered in combination with these drugs 
octreotide plus albumin in a randomized trial comprising 49 patients with cirrhosis and type 1 or 2 HRS. There was a significantly higher rate of recovery of renal function in patients randomized to the terlipressin plus albumin group than in patients randomized to the oral midodrine and octreotide plus albumin group (70.4 vs. $28.6 \% ; P=0.01$ ).

Reporting of AEs in patients with HRS treated with vasoactive drugs varies between studies. In a meta-analysis by Gifford et al. [20], there were fewer ischemic AEs with noradrenaline, although sample sizes were small in published studies. In the REVERSE study, the safety profile of terlipressin generally was consistent with previous clinical studies and included AEs expected with vasoactive drugs. The overall AE rate did not differ between the terlipressin and placebo groups. Gastrointestinal events, most commonly abdominal pain and diarrhea, were the most frequent treatment-related AEs. Ischemic events were more common in patients receiving terlipressin, as was expected [76]. Tailoring treatment, such as modifying the dose and the method of administration, can help reduce these side effects.

\section{Optimal Dosage Regimen of Terlipressin}

Current guidelines recommend that terlipressin $1 \mathrm{mg}$ should be given every $4-6 \mathrm{~h}$ by intravenous bolus injection, in combination with albumin [72]. The aim of treatment is to improve renal function by decreasing $\mathrm{sCr}<133 \mu \mathrm{mol} / \mathrm{L}$ $(<1.5 \mathrm{mg} / \mathrm{dL})$, which indicates complete response [72]. In some countries, a lower initial dose of terlipressin is given in daily practice $(0.5 \mathrm{mg})$ [16]. This dose has been found to be as effective as $1 \mathrm{mg}$ in clinical practice, but benefits from fewer side effects.

In a RCT, Cavallin et al. [86] demonstrated that terlipressin given by continuous infusion was better tolerated than intravenous boluses in the treatment of type $1 \mathrm{HRS}$ and presented fewer side effects ( 35.3 vs. $62.2 \% ; P<0.025$ ). Terlipressin was also effective at doses lower than those required for intravenous bolus administration. The rate of response to treatment, including both complete and partial response, was not significantly different between the two groups (76.5 vs. $64.9 \%)$.

\section{Recommendations}

- Terlipressin can be given as a continuous intravenous infusion (Grade B).

- Bolus administration of terlipressin, which was originally tested and recommended, can also be considered, although with more side effects and similar effectiveness (Grade A).

- Patients who meet diagnostic criteria of HRS-AKI should be given terlipressin $1 \mathrm{mg}$ every $4-6 \mathrm{~h}$ by intravenous bolus injection, when available and there is no contrain- dication, in combination with albumin (Grade A). Terlipressin can be administered at a lower initial dose of $0.5 \mathrm{mg}$ in type $1 \mathrm{HRS}$ (Grade C).

- Terlipressin should be used with caution in patients with underlying severe cardiovascular or peripheral arterial disease. Patients should be closely monitored for cardiovascular or ischemic side effects (Grade A).

\section{Acute on Chronic Liver Failure and Type 1 HRS}

Acute on chronic liver failure (ACLF) is a recognized syndrome that develops in patients with cirrhosis and is characterized by acute decompensation of cirrhosis, single or multiple organ failures, severe systemic inflammation and high short-term mortality [87]. AKI is one of the defining features of ACLF and is important for grading the severity of ACLF [88]. ACLF is graded into three stages: ACLF-1 = single renal failure or single non-renal organ failure if associated with renal dysfunction and/or cerebral dysfunction; ACLF-2 = two organ failures; and ACLF- $3=$ three to six organ failures, with increasing 28-day mortality rate [89]. In order that appropriate and early management can be initiated, it is important to distinguish between non-HRS-AKI and HRS-AKI when reviewing patients with ACLF and AKI [88]. Terlipressin infusion provides earlier response (Day 7: 41.7 vs. $20.0 \% ; P=0.01)$, greater HRS reversal ( 40.0 vs. $16.7 \% ; P=0.004)$ and improved survival (28-day: 48.3 vs. $20.0 \% ; P=0.001)$ compared to noradrenaline in ACLF patients with HRS-AKI [90].

Terlipressin and albumin are effective treatments for type 1 HRS; however, the effects of ACLF grade on response to treatment are not clear [91]. In a recent retrospective analysis of four different cohorts of consecutive patients with HRS treated with terlipressin and albumin, ACLF grade was the largest determinant of response. Of patients with grade 1 ACLF, $60 \%$ responded to treatment; among those with grade 2 ACLF, $48 \%$ responded, and among those with grade 3 ACLF, 29\% responded $(P<0.001$ for comparison between grades) [91].

\section{Recommendations}

- Treatment with terlipressin in patients with ACLF and HRS is associated with an improvement in kidney function and should be started as soon as possible after the diagnosis of type 1 HRS. The success depends on the grade of ACLF (Grade B).

\section{Type 2 HRS}

There is limited evidence for the use of vasoactive drugs in patients who have type 2 HRS. Some trials combined patients with type 1 and type 2 HRS, but the sample sizes 
were small. Two studies evaluated the use of terlipressin in this patient population $[92,93]$. In a randomized study of 46 patients with cirrhosis and HRS, improvement of renal function was more frequent in patients with type 2 HRS compared with that of patients with type 1 HRS (67 vs. 35\%, respectively) treated with terlipressin and albumin, although the difference was not statistically significant [92].

\section{Recommendations}

- There is limited evidence for the use of terlipressin in combination with albumin for patients who have cirrhosis and type 2 HRS, although it has been shown to be effective in clinical practice (Grade C).

- Further prospective studies are needed to assess the efficacy of terlipressin in patients with cirrhosis and type 2 HRS (Grade D).

\section{Conclusions}

Variceal bleeding and HRS are severe complications of cirrhosis associated with significant morbidity and mortality. Data have shown that early diagnosis and intervention improve patients' prognosis. The primary goals of treatment for BEV are to control the acute hemorrhage, prevent early rebleeding and reduce mortality. Vasoactive drugs are used as initial treatment of BEV before endoscopy. Evidence from a multitude of clinical trials and meta-analyses comparing different vasoactive drugs suggests near equivalence in efficacy in terms of initial hemostasis. With terlipressin, mortality benefit was observed. In order to further improve the tolerability of terlipressin, the use of continuous intravenous infusion is currently being explored.

The primary goal of pharmacological therapy in HRS is normalization of renal function. The revised ICA HRSAKI criteria allow physicians to initiate potentially effective treatment, earlier, which might further improve the survival of these patients. The administration of vasoactive drugs (terlipressin, noradrenaline and midodrine plus octreotide) in combination with albumin is currently considered the treatment of choice. In this regard, terlipressin significantly improves reversal of HRS compared to placebo.

Acknowledgment In China, this study was supported by the State Key Projects Specialized on Infectious Diseases (2017ZX10203202-004) and Beijing Municipal Administration of Hospitals Clinical Medicine Development of Special Funding (ZYLX201610) for Dr Huiguo Ding.

Funding The study was sponsored by Ferring Pharmaceuticals. The manuscript was written by Strategen Ltd, who received funding from Ferring Pharmaceuticals in this regard. All named authors meet the International Committee of Medical Journal Editors (ICMJE) criteria for authorship for this manuscript, take responsibility for the integrity of the work as a whole and have given final approval to the version to be published. Ferring Pharmaceuticals reviewed the manuscript but editorial control rested solely with the authors.

\section{Compliance with ethical standards}

Conflicts of interest Frederik Nevens has received a consultancy grant from Ferring. Manuel Mendizabal has received payments for lectures from Bristol-Myers Squibb, AbbVie, Gilead, Ferring and Bayer; honoraria for consulting from Ferring and Gilead. Paolo Angeli declares the following: 2014-2018: Sequana Medical AG Advisory Board; 2016-2018: Biovie Advisory Board and patent application; 2016 Gilead (Italy): Advisory board and grant; 2014 Bhering: travel grant; 2016 Kedrion: speaker invitation. Paulo Lisboa Bittencourt, Minneke J Coenraad, Huiguo Ding, Ming-Chih Hou, Pierre-François Laterre, Nayeli Xochiquetzal Ortiz-Olvera, Julio D. Vorobioff, Wenhong Zhang declare that they have no conflict of interest.

\section{References}

1. Scalone L, Fagiuoli S, Ciampichini R, et al. The societal burden of chronic liver diseases: results from the COME study. $B M J$ Open Gastroenterol. 2015;2:e000025.

2. Neff GW, Duncan CW, Schiff ER. The current economic burden of cirrhosis. Gastroenterol Hepatol (N Y). 2011;7:661-671.

3. Scaglione S, Kliethermes S, Cao G, et al. The epidemiology of cirrhosis in the United States: a population-based study. J Clin Gastroenterol. 2015;49:690-696.

4. GBD 2013 Mortality and Causes of Death Collaborators. Global, regional, and national age-sex specific all-cause and cause-specific mortality for 240 causes of death, 1990-2013: a systematic analysis for the Global Burden of Disease Study 2013. Lancet. 2015;385:117-171.

5. Zatoński WA, Sulkowska U, Mańczuk M, et al. Liver cirrhosis mortality in Europe, with special attention to Central and Eastern Europe. Eur Addict Res. 2010;16:193-201.

6. LaBrecque D, Khan AG, Sarin SK, LeMair AW. Esophageal Varices. World Gastroenterology Organisation Global Guidelines; 2014.

7. Biecker E. Portal hypertension and gastrointestinal bleeding: diagnosis, prevention and management. World J Gastroenterol. 2013;19:5035-5050.

8. Triantafyllou M, Stanley AJ. Update on gastric varices. World J Gastrointest Endosc. 2014;6:168-175.

9. Wani ZA, Bhat RA, Bhadoria AS, Maiwall R, Choudhury A. Gastric varices: classification, endoscopic and ultrasonographic management. J Res Med Sci. 2015;20:1200-1207.

10. Graham DY, Smith JL. The course of patients after variceal hemorrhage. Gastroenterology. 1981;80:800-899.

11. Nevens F. Novel approaches to reducing the risk of variceal hemorrhage. Dig Dis. 2017;35:397-401.

12. de Franchis. On behalf of the Baveno VI Faculty. Expanding consensus in portal hypertension: report of the Baveno VI consensus workshop: stratifying risk and individualizing care for portal hypertension. J Hepatol. 2015;63:743-752.

13. Garcia-Pagán JC, Caca K, Bureau C, et al. Early TIPS (Transjugular Intrahepatic Portosystemic Shunt) cooperative study group. Early use of TIPS in patients with cirrhosis and variceal bleeding. N Engl J Med. 2010;24:2370-2379.

14. Jairath V, Rehal S, Logan R, et al. Acute variceal haemorrhage in the United Kingdom: patient characteristics, management and outcomes in a nationwide audit. Dig Liver Dis. 2014;46:419-426. 
15. Bucsics T, Krones E. Renal dysfunction in cirrhosis: acute kidney injury and the hepatorenal syndrome. Gastroenterol Rep. 2017;5:127-137.

16. Salerno F, Cazzaniga M, Merli M, et al. Diagnosis, treatment and survival of patients with hepatorenal syndrome: a survey on daily medical practice. J Hepatol. 2011;55:1241-1248.

17. Salerno F, Gerbes A, Ginès P, Wong F, Arroyo V. Diagnosis, prevention and treatment of hepatorenal syndrome in cirrhosis. Gut. 2007;56:1310-1318.

18. Wadei HM, Mai ML, Ashan N, Gonwa TA. Hepatorenal syndrome: pathophysiology and management. Clin J Am Soc Nephrol. 2006;1:1066-1079.

19. Ginès A, Escorsell A, Ginès $\mathrm{P}$, et al. Incidence, predictive factors, and prognosis of the hepatorenal syndrome in cirrhosis with ascites. Gastroenterology. 1993;105:229-236.

20. Gifford FJ, Morling JR, Fallowfield JA. Systematic review with meta-analysis: vasoactive drugs for the treatment of hepatorenal syndrome type 1. Aliment Pharmacol Ther. 2017;45:593-603.

21. Howick J, Chalmers I, Glasziou P, et al. The 2011 Oxford Centre for Evidence-Based Medicine Levels of Evidence: Introductory Document. Oxford Centre for Evidence-Based Medicine. Available at: https://www.cebm.net/wp-content/uploads/2014/06/ CEBM-Levels-of-Evidence-Introduction-2.1.pdf. Accessed August 22, 2018.

22. OCEBM Levels of Evidence Working Group. Oxford Centre for Evidence-Based Medicine-Levels of Evidence (March 2009). Available at: https://www.cebm.net/2009/06/oxford-centr e-evidence-based-medicine-levels-evidence-march-2009/. Accessed August 22, 2018.

23. Tripathi D, Stanley AJ, Hayes PC, et al. UK guidelines on the management of variceal haemorrhage in cirrhotic patients. Gut. 2015;64:1680-1704.

24. Garcia-Tsao G, Abraldes JG, Berzigotti A, Bosch J. Portal hypertensive bleeding in cirrhosis: risk stratification, diagnosis, and management: 2016 practice guidance by the American Association for the study of liver diseases. Hepatology. 2017;65:310-335

25. Bernard B, Grangé JD, Khac EN, Amiot X, Opolon P, Poynard T. Antibiotic prophylaxis for the prevention of bacterial infections in cirrhotic patients with gastrointestinal bleeding: a meta-analysis. Hepatology. 1999;29:1655-1661.

26. Bañares R, Albillos A, Rincón D, et al. Endoscopic treatment versus endoscopic plus pharmacologic treatment for acute variceal bleeding: a meta-analysis. Hepatology. 2002;35:609-615.

27. Wells M, Chande N, Adams P, Beaton M, et al. Meta-analysis: vasoactive medications for the management of acute variceal bleeds. Aliment Pharmacol Ther. 2012;35:1267-1278.

28. Söderlund C, Magnusson I, Törngren S, Lundell L. Terlipressin (triglycyl-lysine vasopressin) controls acute bleeding oesophageal varices. A double-blind, randomized, placebo-controlled trial. Scand J Gastroenterol. 1990;25:622-630.

29. Freeman JG, Cobden I, Record CO. Placebo-controlled trial of terlipressin (glypressin) in the management of acute variceal bleeding. J Clin Gastroenterol. 1989;11:58-60.

30. Walker S, Stiehl A, Raedsch R, Kommerell B. Terlipressin in bleeding esophageal varices: a placebo-controlled, double-blind study. Hepatology. 1986;6:112-115.

31. Levacher S, Letoumelin P, Pateron D, Blaise M, Lapandry C, Pourriat JL. Early administration of terlipressin plus glyceryl trinitrate to control active upper gastrointestinal bleeding in cirrhotic patients. Lancet. 1995;346:865-868.

32. Ioannou GN, Doust J, Rockey DC. Terlipressin for acute esophageal variceal hemorrhage. Cochrane Database Syst Rev. 2003; Art. No: CD002147.

33. Seo YS, Park SY, Kim JH, et al. Lack of difference among terlipressin, somatostatin, and octreotide in the control of acute gastroesophageal variceal hemorrhage. Hepatology. 2014;60:954-963.

34. Abid S, Jafri W, Hamid S, et al. Terlipressin versus octreotide in bleeding esophageal varices as an adjuvant therapy with endoscopic band ligation: a randomized double-blind placebo-controlled trial. Am J Gastroenterol. 2009;104:617-623.

35. Feu F, RuizdelArbol L, Bañares R, Planas R, Bosch J. Doubleblind randomized controlled trial comparing terlipressin and somatostatin for acute variceal hemorrhage. Variceal Bleeding Study Group. Gastroenterology. 1996;111:1291-1299.

36. Walker S, Kreichgauer HP, Bode JC. Terlipressin (glypressin) versus somatostatin in the treatment of bleeding esophageal varices-final report of a placebo-controlled, double-blind study. Z Gastroenterol. 1996;34:692-698.

37. Pedretti G, Elia G, Calzetti C, Magnani G, Fiaccadori F. Octreotide versus terlypressin in acute variceal hemorrhage in liver cirrhosis. Emergency control and prevention of early rebleeding. Clin Investig. 1994;72:653-659.

38. Silvain C, Carpentier S, Sautereau D, et al. Terlipressin plus transdermal nitroglycerin vs. octreotide in the control of acute bleeding from esophageal varices: a multicenter randomized trial. Hepatology. 1993;18:61-65.

39. Bittencourt PL, Farias AQ, Strauss E, et al. Pannel of the 1st Brazilian Consensus of Variceal Bleeding, Brazilian Society of Hepatology. Variceal bleeding: consensus meeting report from the Brazilian Society of Hepatology. Arq Gastroenterol. 2010;47:202-216.

40. Krag A, Borup T, Møller S, et al. Efficacy and safety of terlipressin in cirrhotic patients with variceal bleeding or hepatorenal syndrome. Adv Ther. 2008;25:1105-1140.

41. Sola E, Lens S, Guevara M, et al. Hyponatremia in patients treated with terlipressin for severe gastrointestinal bleeding due to portal hypertension. Hepatology. 2010;52:1783-1790.

42. Yim SY, Seo YS, Jung CH, et al. Risk factors for developing hyponatremia during terlipressin treatment: a retrospective analyses in variceal bleeding. J Clin Gastroenterol. 2015;49:607-612.

43. Ge PS, Runyon BA. The changing role of beta-blocker therapy in patients with cirrhosis. J Hepatol. 2014;60:643-653.

44. Kalambokis G, Economou M, Paraskevi K, et al. Effects of somatostatin, terlipressin and somatostatin plus terlipressin on portal and systemic hemodynamics and renal sodium excretion in patients with cirrhosis. J Gastroenterol Hepatol. 2005;20:1075-1081.

45. Lin HC, Yang YY, Hou MC, et al. Hemodynamic effects of a combination of octreotide and terlipressin in patients with viral hepatitis related cirrhosis. Scand J Gastroenterol. 2002;37:482-487.

46. Nidegger D, Ragot S, Berthelémy P, et al. Cirrhosis and bleeding: the need for very early management. J Hepatol. 2003;39:509-514.

47. Avgerinos A, Nevens F, Raptis S, Fevery J. Early administration of somatostatin and efficacy of sclerotherapy in acute oesophageal variceal bleeds: the European Acute Bleeding Oesophageal Variceal Episodes (ABEVE) randomised trial. Lancet. 1997;350:1495-1499.

48. Calès P, Masliah C, Bernard B, et al. Early administration of vapreotide for variceal bleeding in patients with cirrhosis. French Club for the Study of Portal Hypertension. N Engl J Med. 2001;344:23-28.

49. Escorsell A, Bandi JC, Moitinho E, et al. Time profile of the haemodynamic effects of terlipressin in portal hypertension. $J$ Hepatol. 1997;26:621-627.

50. ClinicalTrials.gov Identifier: NCT0269586, To assess safety and efficacy of bolus versus continuous infusion of terlipressin in acute variceal bleeding. Available at: https://clinicaltrials.gov/ ct2/show/NCT02695862.

51. Chang TT, Lee FY, Tsai YT, et al. A randomized controlled study of low-dose and high-dose terlipressin in the control of acute 
oesophageal variceal haemorrhage. J Gastroenterol Hepatol. 1991;6:481-484.

52. Azam Z, Hamid S, Jafri W, et al. Short course adjuvant terlipressin in acute variceal bleeding: a randomized double blind dummy controlled trial. J Hepatol. 2012;56:819-824.

53. Garcia-Pagán JC, Di Pascoli M, Caca K, et al. Early TIPS (Transjugular Intrahepatic Portosystemic Shunt) Cooperative Study Group. Use of early-TIPS for high-risk variceal bleeding: results of a post-RCT surveillance study. J Hepatol. 2013;58:45-50.

54. Altraif I, Handoo FA, Aljumah A, et al. Effect of erythromycin before endoscopy in patients presenting with variceal bleeding: a prospective, randomized, double-blind, placebo-controlled trial. Gastrointest Endosc. 2011;73:245-250.

55. Brunner F, Berzigotti A, Bosch J. Prevention and treatment of variceal haemorrhage in 2017. Liver Int. 2017;37:104-115.

56. Cipolletta L, Zambelli A, Bianco MA, et al. Acrylate glue injection for acutely bleeding oesophageal varices: a prospective cohort study. Dig Liver Dis. 2009;41:729-734.

57. Ribeiro JP, Matuguma SE, Cheng S, et al. Results of treatment of esophageal variceal hemorrhage with endoscopic injection of $n$-butyl-2-cyanoacrylate in patients with Child-Pugh class C cirrhosis. Endosc Int Open. 2015;3:584-589.

58. Garcia-Tsao G, Sanyal AJ, Grace ND, Carey W. Prevention and management of gastroesophageal varices and variceal hemorrhage in cirrhosis. Hepatology. 2007;46:922-938.

59. Sarin SK, Jain AK, Jain M, Gupta R. A randomized controlled trial of cyanoacrylate versus alcohol injection in patients with isolated fundic varices. Am J Gastroenterol. 2002;97:1010-1015.

60. Lo GH, Lai KH, Cheng JS, Chen MH, Chiang HT. A prospective, randomized trial of butyl cyanoacrylate injection versus band ligation in the management of bleeding gastric varices. Hepatology. 2001;33:1060-1064.

61. Huang YH, Yeh HZ, Chen GH, et al. Endoscopic treatment of bleeding gastric varices by $N$-butyl-2-cyanoacrylate (Histoacryl) injection: long-term efficacy and safety. Gastrointest Endosc. 2000;52:160-167.

62. Lee YT, Chan FK, Ng EK, et al. EUS-guided injection of cyanoacrylate for bleeding gastric varices. Gastrointest Endosc. 2000;52:168-174.

63. Al-Ali J, Pawlowska M, Coss A, Svarta S, Byrne M, Enns R. Endoscopic management of gastric variceal bleeding with cyanoacrylate glue injection: safety and efficacy in a Canadian population. Can J Gastroenterol. 2010;24:593-596.

64. Ríos Castellanos E, Seron P, Gisbert JP, Bonfill Cosp X. Endoscopic injection of cyanoacrylate glue versus other endoscopic procedures for acute bleeding gastric varices in people with portal hypertension. Cochrane Database Syst Rev. 2015; Art. No.: CD010180.

65. Chau TN, Patch D, Chan YW, Nagral A, Dick R, Burroughs AK. "Salvage" transjugular intrahepatic portosystemic shunts: gastric fundal compared with esophageal variceal bleeding. Gastroenterology. 1998;114:981-987.

66. Azoulay D, Castaing D, Majno P, et al. Salvage transjugular intrahepatic portosystemic shunt for uncontrolled variceal bleeding in patients with decompensated cirrhosis. J Hepatol. 2001;35:590-597.

67. Lo GH, Liang HL, Chen WC, et al. A prospective, randomized controlled trial of transjugular intrahepatic portosystemic shunt versus cyanoacrylate injection in the prevention of gastric variceal rebleeding. Endoscopy. 2007;39:679-685.

68. Arroyo V, Ginès $\mathrm{P}$, Gerbes $\mathrm{AL}$, et al. Definition and diagnostic criteria of refractory ascites and hepatorenal syndrome in cirrhosis. International Ascites Club. Hepatology. 1996;23:164-176.

69. Angeli P, Ginès P, Wong F, et al. Diagnosis and management of acute kidney injury in patients with cirrhosis: revised consensus recommendations of the International Club of Ascites. $J$ Hepatol. 2015;62:968-974.

70. Rodriguez E, Elia C, Solà E, et al. Terlipressin and albumin for type-1 hepatorenal syndrome associated with sepsis. J Hepatol. 2014;60:955-961.

71. Boyer TD, Sanyal AJ, Garcia-Tsao G, et al. Predictors of response to terlipressin plus albumin in hepatorenal syndrome (HRS) type 1: relationship of serum creatinine to hemodynamics. J Hepatol. 2011;55:315-321.

72. European Association for the Study of the Liver. EASL clinical practice guidelines on the management of ascites, spontaneous bacterial peritonitis, and hepatorenal syndrome in cirrhosis. $J$ Hepatol. 2010;53:397-417.

73. Garcovich M, Zocco MA, Gasbarrini A. Clinical use of albumin in hepatology. Blood Transfus. 2009;7:268-277.

74. Dobre M, Demirjian S, Sehgal AR, Navaneethan SD. Terlipressin in hepatorenal syndrome: a systematic review and meta-analysis. Int Urol Nephrol. 2011;43:175-184.

75. Fabrizi F, Dixit V, Messa P, Martin P. Terlipressin for hepatorenal syndrome: a meta-analysis of randomized trials. Int J Artif Organs. 2009;32:133-140.

76. Boyer TD, Sanyal AJ, Wong F, et al. For REVERSE Study Investigators. Terlipressin plus albumin is more effective than albumin alone in improving renal function in patients with cirrhosis and hepatorenal syndrome type 1. Gastroenterology. 2016;150:15791589.e2.

77. Sanyal AJ, Boyer TD, Frederick RT, et al. Reversal of hepatorenal syndrome type 1 with terlipressin plus albumin versus placebo plus albumin in a pooled analysis of the OT-0401 and REVERSE randomised clinical studies. Aliment Pharmacol Ther. 2017;45:1390-1402.

78. Gluud LL, Christensen K, Christensen E, Krag A. Terlipressin for hepatorenal syndrome. Cochrane Database Syst Rev. 2012;9:CD005162.

79. Martín-Llahí M, Pepin MN, Guevara M, et al. Terlipressin and albumin vs albumin in patients with cirrhosis and hepatorenal syndrome: a randomized study. Gastroenterology. 2008;134:1352-1359.

80. Neri S, Pulvirenti D, Malaguarnera M, et al. Terlipressin and albumin in patients with cirrhosis and type I hepatorenal syndrome. Dig Dis Sci. 2008;53:830-835. https://doi.org/10.1186/s4006 4-015-1625-z.

81. Goyal O, Sidhu SS, Sehgal N, Puri S. Noradrenaline is as effective as terlipressin in hepatorenal syndrome type 1: a prospective, randomized trial. J Assoc Phys India. 2016;64:30-35.

82. Singh V, Ghosh S, Singh B, et al. Noradrenaline vs. terlipressin in the treatment of hepatorenal syndrome: a randomized study. $J$ Hepatol. 2012;56:1293-1298.

83. Sharma P, Kumar A, Shrama BC, Sarin SK. An open label, pilot, randomized controlled trial of noradrenaline versus terlipressin in the treatment of type 1 hepatorenal syndrome and predictors of response. Am J Gastroenterol. 2008;103:1689-1697.

84. Alessandria C, Ottobrelli A, Debernardi-Venon W, et al. Noradrenalin versus terlipressin in patients with hepatorenal syndrome: a prospective, randomized, unblinded, pilot study. J Hepatol. 2007;47:499-505.

85. Cavallin M, Kamath PS. Italian Association for the Study of the Liver Study Group on Hepatorenal Syndrome. Terlipressin plus albumin versus midodrine and octreotide plus albumin in the treatment of hepatorenal syndrome: a randomized trial. Hepatology. 2015;62:567-574.

86. Cavallin M, Piano S, Romano A, et al. Terlipressin given by continuous intravenous infusion versus intravenous boluses in the treatment of hepatorenal syndrome: a randomized controlled study. Hepatology. 2016;63:983-992. 
87. Arroyo V, Moreau R, Kamath PS, et al. Acute-on-chronic liver failure in cirrhosis. Nat Rev Dis Primers. 2016;2:16041.

88. Davenport A, Sheikh MF, Lamb E, Agarwal B, Jalan R. Acute kidney injury in acute-on-chronic liver failure: Where does hepatorenal syndrome fit? Kidney Int. 2017;92:1058-1070.

89. Arroyo V, Jalan R. Acute-on-chronic liver failure: definition, diagnosis, and clinical characteristics. Semin Liver Dis. 2016;36:109-116.

90. Arora V, Maiwall R, Vijayaraghavan R, et al. Terlipressin is superior to noradrenaline in the management of acute kidney injury in acute on chronic liver failure. Hepatology. 2018;. https://doi. org/10.1002/hep.30208.
91. Piano S, Schmidt HH, Ariza X, et al. Association between grade of acute on chronic liver failure to terlipressin and albumin in patients with hepatorenal syndrome. Clin Gastroenterol Hepatol. 2018;. https://doi.org/10.1016/j.cgh.2018.01.035.

92. Martín-Llahí M, Pepin MN, Guevara M, et al. Terlipressin and albumin vs albumin in patients with cirrhosis and hepatorenal syndrome: a randomized study. Gastroenterology. 2008;134:1352-1359.

93. Alessandria C, Venon WD, Marzano A, Barletti C, Fadda M, Rizzetto M. Renal failure in cirrhotic patients: role of terlipressin in clinical approach to hepatorenal syndrome type 2. Eur J Gastroenterol Hepatol. 2002;14:1363-1368.

\section{Affiliations}

\section{Frederik Nevens ${ }^{1}$ (D) Paulo Lisboa Bittencourt ${ }^{2} \cdot$ Minneke J. Coenraad $^{3} \cdot$ Huiguo Ding ${ }^{4} \cdot$ Ming-Chih Hou $^{5}$. Pierre-François Laterre ${ }^{6} \cdot$ Manuel Mendizabal $^{7} \cdot$ Nayeli Xochiquetzal Ortiz-Olvera $^{8}$. Julio D. Vorobioff ${ }^{9}$. Wenhong Zhang $^{10} \cdot$ Paolo Angeli ${ }^{11}$}

Paulo Lisboa Bittencourt plbbr@uol.com.br

Minneke J. Coenraad

M.J.Coenraad@lumc.nl

Huiguo Ding

dinghuiguo@ccmu.edu.cn

Ming-Chih Hou

mchou@vghtpe.gov.tw

Pierre-François Laterre

pierre-francois.laterre@uclouvain.be

Manuel Mendizabal

MMENDIZA@cas.austral.edu.ar

Nayeli Xochiquetzal Ortiz-Olvera

nayelixoortiz@yahoo.com.mx

Julio D. Vorobioff

jdvorobioff@hotmail.com

Wenhong Zhang

zhangwhc@163.com

Paolo Angeli

paolo.angeli54@gmail.com

1 Department of Gastroenterology and Hepatology, University Hospitals Leuven, KU Leuven, Campus Gasthuisberg, Herestraat 49, 3000 Louvain, Belgium

2 Unit of Gastroenterology and Hepatology, Portuguese Hospital of Salvador, Rua Prof. Clementino Fraga, 220/1901, Salvador, Bahia, Brazil
3 Department of Gastroenterology and Hepatology, Leiden University Medical Center, Albinusdreef 2, 2300 ZC Leiden, The Netherlands

4 Department of Gastroenterology and Hepatology, Beijing You' an Hospital Affiliated with Capital Medical University, No 8, Youan Men Wai Street, Fengtai District, Beijing 100069, China

5 Department of Medicine Division of Gastroenterology, Taipei Veterans General Hospital, 201, Sec. II, Shih-Pai Road, Taipei, Taiwan

6 Medical-surgical Intensive Care Unit, Cliniques Universitaires Saint Luc, Université Catholique de Louvain, Avenue Hippocrate 10, Brussels, Belgium

7 Hepatology and Liver Transplant Unit, Hospital Universitario Austral, Av. Peron 1500, 1629 Pilar, Provincia de Buenos Aires, Argentina

8 Department of Gastroenterology, UMAE, Hospital de Especialidades Dr. Bernardo Sepúlveda, Centro Médico Nacional Siglo XXI, IMSS, Mexico City, Mexico

9 Department of Gastroenterology and Hepatology, University of Rosario Medical School, Morrison 8750, 2000 Rosario, Argentina

10 Department of Infectious Diseases, Huashan Hospital, Fudan University, 12 Wulumuqi Middle Rd, Jingan Qu, Shanghai Shi 200000, China

11 Unit of Internal Medicine and Hepatology (UIMH), Department of Medicine - DIMED, University of Padova, Via Giustiniani, 2, 35128 Padua, Italy 